\title{
Complete Sequence of the Mitochondrial Genome of Spirometra ranarum: Comparison with S. erinaceieuropaei and S. decipiens
}

\author{
Hyeong-Kyu Jeon, Hansol Park, Dongmin Lee, Seongjun Choe, Yeseul Kang, Mohammed Mebarek Bia, \\ Sang-Hwa Lee, Keeseon S. Eom* \\ Department of Parasitology, Parasite Research Center and Parasite Resource Bank, Chungbuk National University School of Medicine, \\ Cheongju 28644, Korea
}

\begin{abstract}
This study was undertaken to determine the complete mitochondrial DNA sequence and structure of the mitochondrial genome of Spirometra ranarum, and to compare it with those of S. erinaceieuropaei and S. decipiens. The aim of this study was to provide information of the species level taxonomy of Spirometra spp. using the mitochondrial genomes of 3 Spirometra tapeworms. The S. ranarum isolate originated from Myanmar. The mitochondrial genome sequence of S. ranarum was compared with that of S. erinaceieuropaei (GenBank no. KJ599680) and S. decipiens (GenBank no. KJ599679). The complete mtDNA sequence of $S$. ranarum comprised 13,644 bp. The $S$. ranarum mt genome contained 36 genes comprising 12 protein-coding genes, 22 tRNAs and 2 rRNAs. The mt genome lacked the atp8 gene, as found for other cestodes. All genes in the $S$. ranarum mitochondrial genome are transcribed in the same direction and arranged in the same relative position with respect to gene loci as found for S. erinaceieuropaei and S. decipiens mt genomes. The overall nucleotide sequence divergence of 12 protein-coding genes between $\mathrm{S}$. ranarum and $\mathrm{S}$. decipiens differed by $1.5 \%$, and $100 \%$ sequence similarity was found in the cox 2 and nad 6 genes, while the DNA sequence divergence of the cox 1 , nad1, and nad4 genes of $S$. ranarum and S. decipiens was $2.2 \%, 2.1 \%$, and $2.6 \%$, respectively.
\end{abstract}

Key words: Spirometra ranarum, Spirometra erinaceieuropaei, Spirometra decipiens, mitochondrial genome

Cestodes of the genus Spirometra (Mueller, 1934) are intestinal parasites of cats and dogs that use the fresh water copepod as the first intermediate host and reptiles or amphibians as the second intermediate host. Spirometra species have been reported from felids and canids worldwide under the generic name Diphyllobothrium since 19th century. Spirometra species were described from the morphological identification of 6 Spirometra species, comprising S. erinaceieuropaei (Rudolphi, 1819), S. decipiens (Diesing, 1850), S. ranarum (Gastaldi, 1854), S. mansoni (Cobbold, 1882), S. houghtoni (Syn. S. mansoni), and S. okumurai by Faust et al. [1]. Fifteen Spirometra species were reviewed as valid species and divided into 2 groups by Wardle and McLeod [2]. A Spirometra species in North America was reported as S. mansonoides (McIntosh, 1935) [3]. Five Spirometra

\footnotetext{
- Received 11 November 2018, revised 23 January 2019, accepted 28 January 2019.

*Corresponding author (kseom@chungbuk.ac.kr)

(c) 2019, Korean Society for Parasitology and Tropical Medicine

This is an Open Access article distributed under the terms of the Creative Commons Attribution Non-Commercial License (http://creativecommons.org/licenses/by-nc/4.0) which permits unrestricted non-commercial use, distribution, and reproduction in any medium, provided the original work is properly cited.
}

species have been reported from wild felids in South America such as S. decipiens, S. mansoni, S. longicollis (Parodi and Widakowich, 1917), S. gracilis (Baer, 1927), and S. mansonoides [4]. S. pretoriensis and $S$. theileri (Baer, 1924) have been reported from a bush cat (Leptailurus serval) and a tiger cat (Felis lybica cafra) [5]. Currently, Spirometra species causing human sparganosis have been identified as $S$. erinaceieuropaei and $S$. decipiens by morphological and genetic analyses in Korea [6]. The most recent reports include identification of $S$. ranarum from frogs (Hoplobatrachus rugulosus) in Myanmar and from lions (Panthera leo) in Tanzania by analysis of mitochondrial genes and morphological observation $[7,8]$. S. ranarum obtained from cats and dogs were identified by molecular and phylogenetic analysis of DNA sequence data of the mitochondrial cytochrome $c$ oxidase ( $\operatorname{cox} 1$ ) gene in Korea [9].

A species level taxonomy of Spirometra species was employed based on morphological characteristics combined with molecular analysis. Mitochondrial DNA is considered to provide a useful molecular marker for taxonomic identification, inferences of phylogenetic relationships, population genetics and 
epidemiological investigation [10]. Mitochondrial DNA sequences have been studied for genetic variation, taxonomy, and phylogenetic relationships using cox1, cox3, nad1, nad3, and nad4 [10-14]. The complete mitochondrial genomes of order Diphyllobothridea have been published for D. latum, D. nihonkaiense, S. erinaceieuropaei, and S. decipiens [15-17]. This study was undertaken to determine the complete mitochondrial DNA sequence and structure of the mitochondrial genome of $S$. ranarum, and to compare it with those of $S$. erinaceieuropaei and S. decipiens. The aim of this study was to provide information of the species level taxonomy of Spirometra spp., by analysis of mitochondrial genomes of 3 Spirometra tapeworms.

The S. ranarum isolate originated from Myanmar. This Spirometra tapeworm was identified using morphological and molecular characteristics as well as animal inoculation experiments [7]. Specific identity was confirmed using sequences of the mitochondrial cox1 and nad1 genes previously reported for $S$. ranarum (GenBank No. MH298843, MH2998844). Mitochondria from a single specimen were isolated using a Qproteome Mitochondria Isolation Kit (Qiagen, Hilden, Germany). Mitochondrial DNA (mtDNA) was extracted from mitochondria using a DNeasy Blood and Tissue Kit (Qiagen, Valencia, California, USA). Whole genome amplification (WGA) of extracted mtDNA was performed using an REPLI-g Mitochondrial DNA Kit (Qiagen, Hilden, Germany) combined with ExonucleaseResistant Random Primer: 5'NpNpNpNpNpSNpSN-3' (Thermo Scientific, Hudson, New Hampshire, USA). Amplified mtDNA was sequenced using an Illumina sequencing genome analyzer (Macrogen, Seoul, Korea). The mitogenome was reconstructed using the Geneious 9.0 software program (Biomatters Ltd, Auckland, New Zealand) and employing the partial cox1 gene of $S$. ranarum as initial bait. The complete mitochondrial genome was annotated and recovered using MITOS and Dogma $[18,19]$. The mitochondrial genome sequence of $S$. ranarum was compared with that of S. erinaceieuropaei (GenBank no. KJ599680) and S. decipiens (GenBank no. KJ599679). The 12 protein-encoding genes in the mtDNA was confirmed by comparison with mitochondrial gene sequences of other cestodes available in the GenBank database. Platyhelminth mitochondrial genetic code was used to translate the mitochondrial protein-coding genes. Twenty-two putative tRNA genes were identified using tRNAscan-SE.2.1 [20] and anticodon sequences. The putative stem-loop structures of non-coding regions were inferred using the RNAdraw program [21].

The complete mtDNA sequence of $S$. ranarum comprised
$13,644 \mathrm{bp}$. The S. ranarum mt genome contained 36 genes comprising 12 protein-coding genes, 22 tRNAs and 2 rRNAs. The mt genome lacked the atp 8 gene as with other cestodes. All genes in the S. ranarum mitochondrial genome are transcribed in the same direction and arranged in the same relative position with respect to gene loci as found for $S$. erinaceieuropaei and $S$. decipiens $\mathrm{mt}$ genomes (Table 1). The nucleotide composition of the entire mt genome of $S$. ranarum comprised $20.4 \% \mathrm{~A}, 11.2 \% \mathrm{C}, 22.6 \% \mathrm{G}$, and $45.8 \% \mathrm{~T}(66.2 \% \mathrm{~A}+\mathrm{T})$. An $\mathrm{A}+\mathrm{T}$ richness was observed in Spirometra species $\mathrm{S}$. erinaceieuropaei $(65.7 \% \mathrm{~A}+\mathrm{T})$ and $S$. decipiens $(66.3 \% \mathrm{~A}+\mathrm{T})$ as with other cestodes. Some genes were found to overlap in the mitochondrial genome of $S$. ranarum: cox1/trnT (10 bp), nad4L/nad4 (39 bp), $\operatorname{trnQ} / \operatorname{trnF}$ (3 bp), and trnF/trnM (3 bp) (Table 1).

Accounting for some $74 \%$ of the entire mitochondrial genomes of Spirometra species are protein-coding genes. All putative open reading frames (ORFs) of the 12 protein-coding genes of $S$. ranarum mtDNA started and ended with complete codons. The ATG initiation codon was used in 11 genes (atp6, cob, coxl, cox2, nad1, nad2, nad3, nad4, nad4L, nad5, and nad6), while the GTG initiation codon was used only in the cox3 gene in $S$. ranarum, S. decipiens and S. erinaceieuropaei. The TAG stop codon was used in 4 genes (cox1, nad2, nad4, and nad4L), while the TAA stop codon was used in 6 genes (atp6, cob, cox2, nad1, nad5, and nad6) in S. ranarum and S. decipiens. The TAG codon was used in the cox 2 gene in S. erinaceieuropaei. In cox3 and nad3, the abbreviated stop codon $U$ was confirmed in $S$. ranarum and $S$. decipiens. The use of TA termination was found in $\operatorname{cox} 3$ for $S$. erinaceieuropaei mtDNAs. The most commonly used codons were for leucine, phenylalanine, valine and serine in mitochondrial proteins of 3 Spirometra species. The mitochondrial genome of Spirometra tapeworms appear to use the flatworm mitochondrial code, namely TTR and CTN for leucine, TTY for phenylalanine, GTN for valine, and AGN and TCN for serine.

Twenty-two tRNA genes were identified as putative secondary structures comprising a typical cloverleaf shape, with length ranging from 56 to $69 \mathrm{bp}$ in S. ranarum and S. decipiens, respectively, and 56 to $70 \mathrm{bp}$ in S. erinaceieuropaei. The inferred secondary structure of 19 tRNA exhibited a typical cloverleaf shape with 4 arms comprising aminoacyl acceptor arms, a DHU arm, anticodon stems and TYC arms. trnR, trnS1, and trnS2 were replaced with 7-12 bp of unpaired loop in the DHU arms. The aminoacyl acceptor arms comprising $7 \mathrm{nt}$ such as $\operatorname{trnA}$, trnI, $\operatorname{trnM}, \operatorname{trn} \mathrm{Q}, \operatorname{trnR}, \operatorname{trnS2} 2 \mathrm{trnT}$, and $\operatorname{trn} V$ contained 1 or 3 non-canonical base pairs. The anticodon stems comprising $5 \mathrm{nt}$ as 
Table 1. Position and characteristics of the protein-coding and non-coding sequences in the mt genome of Spirometra ranarum, S. decipiens and S. erinaceieuropaei

\begin{tabular}{|c|c|c|c|c|c|c|c|c|c|c|c|c|}
\hline \multirow{3}{*}{ Genes } & \multicolumn{6}{|c|}{ Length of gene and sequence } & \multicolumn{6}{|c|}{ Codon used for } \\
\hline & \multicolumn{3}{|c|}{ Nucleotide } & \multicolumn{3}{|c|}{ Amino acid } & \multicolumn{3}{|c|}{ Initiation } & \multicolumn{3}{|c|}{ Termination } \\
\hline & $\mathrm{Sr}$ & $\mathrm{Sd}$ & Se & $\mathrm{Sr}$ & $\mathrm{Sd}$ & $\mathrm{Se}$ & $\mathrm{Sr}$ & $\mathrm{Sd}$ & Se & $\mathrm{Sr}$ & $\mathrm{Sd}$ & $\mathrm{Se}$ \\
\hline $\operatorname{trn} G$ & 67 & 67 & 67 & & & & & & & & & \\
\hline $\cos 3^{*}$ & 643 & 643 & 644 & 214 & 214 & 214 & GTG & GTG & GTG & T & $\mathrm{T}$ & TA \\
\hline $\operatorname{trnH}$ & 69 & 69 & 70 & & & & & & & & & \\
\hline$c o b$ & 1,110 & 1,110 & 1,110 & 370 & 370 & 370 & ATG & ATG & ATG & TAA & TAA & TAA \\
\hline nad4L & 261 & 261 & 261 & 87 & 87 & 87 & ATG & ATG & ATG & TAG & TAG & TAG \\
\hline nad4 & 1,254 & 1,254 & 1,254 & 418 & 418 & 418 & ATG & ATG & ATG & TAG & TAG & TAG \\
\hline $\operatorname{trn} Q$ & 64 & 64 & 64 & & & & & & & & & \\
\hline $\operatorname{trnF}$ & 64 & 64 & 64 & & & & & & & & & \\
\hline $\operatorname{trnM}$ & 68 & 68 & 68 & & & & & & & & & \\
\hline atp6 & 516 & 516 & 516 & 172 & 172 & 172 & ATG & ATG & ATG & TAA & TAA & TAA \\
\hline nad2 & 873 & 873 & 873 & 291 & 291 & 291 & ATG & ATG & ATG & TAG & TAG & TAG \\
\hline $\operatorname{trnV}$ & 65 & 65 & 65 & & & & & & & & & \\
\hline $\operatorname{trn} A$ & 61 & 61 & 61 & & & & & & & & & \\
\hline $\operatorname{trn} D$ & 64 & 64 & 66 & & & & & & & & & \\
\hline nad1 & 891 & 891 & 891 & 297 & 297 & 297 & ATG & ATG & ATG & TAA & TAA & TAA \\
\hline $\operatorname{trnN}$ & 66 & 66 & 66 & & & & & & & & & \\
\hline $\operatorname{trn} P$ & 65 & 65 & 65 & & & & & & & & & \\
\hline trnl & 66 & 64 & 64 & & & & & & & & & \\
\hline trnK & 63 & 63 & 63 & & & & & & & & & \\
\hline nad3* & 346 & 346 & 346 & 115 & 115 & 115 & ATG & ATG & ATG & $\mathrm{T}$ & $\mathrm{T}$ & $\mathrm{T}$ \\
\hline trnS1 (AGN) & 59 & 59 & 59 & & & & & & & & & \\
\hline $\operatorname{trnW}$ & 66 & 66 & 65 & & & & & & & & & \\
\hline $\operatorname{cox} 1$ & 1,566 & 1,566 & 1,566 & 522 & 522 & 522 & ATG & ATG & ATG & TAG & TAG & TAG \\
\hline $\operatorname{trn} T$ & 70 & 70 & 69 & & & & & & & & & \\
\hline$r r n L$ & 972 & 973 & 967 & & & & & & & & & \\
\hline $\operatorname{trnC}$ & 65 & 65 & 65 & & & & & & & & & \\
\hline$r r n S$ & 730 & 730 & 733 & & & & & & & & & \\
\hline $\operatorname{cox} 2$ & 570 & 570 & 570 & 190 & 190 & 190 & ATG & ATG & ATG & TAA & TAA & TAG \\
\hline $\operatorname{trn} E$ & 65 & 65 & 65 & & & & & & & & & \\
\hline nad6 & 468 & 468 & 465 & 156 & 156 & 155 & ATG & ATG & ATG & TAA & TAA & TAA \\
\hline $\operatorname{trn} Y$ & 68 & 68 & 68 & & & & & & & & & \\
\hline NR1 & 204 & 204 & 201 & & & & & & & & & \\
\hline $\operatorname{trnL1}(C U N)$ & 67 & 67 & 67 & & & & & & & & & \\
\hline trnS2 (UGN) & 65 & 66 & 66 & & & & & & & & & \\
\hline $\operatorname{trn} L 2$ (UUN) & 65 & 65 & 65 & & & & & & & & & \\
\hline $\operatorname{trnR}$ & 57 & 57 & 56 & & & & & & & & & \\
\hline nad5 & 1,569 & 1,569 & 1,569 & 523 & 523 & 523 & ATG & ATG & ATG & TAA & TAA & TAA \\
\hline NR2 & 175 & 175 & 185 & & & & & & & & & \\
\hline
\end{tabular}

Sr: Spirometra ranarum; Sd: S. decipiens; Se: S. erinaceieuropaei; NR1: non-coding region 1; NR2: long non-coding region 2.

*TAA stop codon is completed by the addition of 3 ' A residues to the mRNA.

with typical stem structures. The TYC arms comprised a 2-5 nt stem with a 3-9 nt loop. The variable loop between the anticodon and the TYC stems comprised 3-5 nt in 3 Spirometra species mtDNA. These 22 tRNAs had the same structure in the mitochondrial genome of other parasitic platyhelminths. Two mitochondrial ribosomal subunit genes $r r n L$ and $r r n S$ in the 3
Spirometra tapeworms were separated by trnC. The putative $16 \mathrm{~S}$ rRNA and 12S rRNA genes in S. ranarum were 972 and $730 \mathrm{nt}$ long, respectively. These sizes are similar to those of rRNA genes in S. decipiens and S. erinaceieuropaei, which range from 967 to $973 \mathrm{nt}$ for 16S rRNA, and 730 to $733 \mathrm{nt}$ for 12S rRNA (Table 1). The nucleotide content of the 16S rRNA and 12S 
Table 2. Divergences of nucleotides and amino acids of the protein-coding genes

\begin{tabular}{|c|c|c|c|c|c|c|c|c|c|c|c|c|c|c|c|}
\hline $\operatorname{cox} 1$ & $\mathrm{Sr}$ & $\mathrm{Sd}$ & $\mathrm{Se}$ & cox2 & $\mathrm{Sr}$ & $\mathrm{Sd}$ & $\mathrm{Se}$ & $\operatorname{cox} 3$ & $\mathrm{Sr}$ & $\mathrm{Sd}$ & $\mathrm{Se}$ & $c o b$ & $\mathrm{Sr}$ & $\mathrm{Sd}$ & Se \\
\hline $\mathrm{Sr}$ & - & 2.2 & 8.8 & & - & 0.0 & 10.4 & & - & 1.4 & 12.7 & & - & 2.4 & 11.2 \\
\hline $\mathrm{Sd}$ & 0.4 & - & 9.4 & & 0.0 & - & 10.4 & & 0.9 & - & 12.0 & & 1.1 & - & 10.9 \\
\hline Se & 2.9 & 2.9 & - & & 3.2 & 3.2 & - & & 5.1 & 5.6 & - & & 3.8 & 4.1 & - \\
\hline
\end{tabular}

\begin{tabular}{|c|c|c|c|c|c|c|c|c|c|c|c|c|}
\hline atp6 & & & & nad1 & & & nad2 & & & nad3 & & \\
\hline $\mathrm{Sr}$ & - & 1.9 & 14.1 & - & 2.1 & 10.0 & - & 1.7 & 14.0 & - & 1.7 & 13.6 \\
\hline $\mathrm{Sd}$ & 1.2 & - & 13.4 & 0.7 & - & 9.8 & 1.0 & - & 13.7 & 0.9 & - & 13.0 \\
\hline Se & 8.2 & 8.2 & - & 5.4 & 6.1 & - & 7.6 & 8.6 & - & 7.8 & 7.0 & - \\
\hline
\end{tabular}

\begin{tabular}{|c|c|c|c|c|c|c|c|c|c|c|c|c|}
\hline \multicolumn{4}{|l|}{ nad4 } & \multicolumn{3}{|l|}{ nad $4 \mathrm{~L}$} & \multicolumn{3}{|l|}{ nad5 } & \multicolumn{3}{|l|}{ nad6 } \\
\hline $\mathrm{Sr}$ & - & 2.6 & 13.7 & - & 1.2 & 11.5 & - & 1.4 & 18.1 & - & 0.0 & 18.8 \\
\hline $\mathrm{Sd}$ & 1.4 & - & 14.0 & 0.2 & - & 11.9 & 0.8 & - & 18.1 & 0.0 & - & 18.8 \\
\hline $\mathrm{Se}$ & 9.1 & 9.4 & - & 2.3 & 2.3 & - & 12.1 & 11.9 & - & 14.8 & 14.8 & - \\
\hline
\end{tabular}

Percentage pairwise divergences of nucleotides (above diagonal) and amino acids (below diagonal) of the 12 protein-coding genes of the Spirometra tapeworms (Sr: Spirometra ranarum [present study], Sd: S. decipiens [KJ599679], Se: S. erinaceieuropaei [KJ599680]).

rRNA genes in S. ranarum was $25.0 \%$ A, $12.5 \%$ C, $24.3 \% \mathrm{G}$ and $38.1 \% \mathrm{~T}(63.1 \% \mathrm{~A}+\mathrm{T})$, whereas the $\mathrm{A}+\mathrm{T}$ content was $63.1 \%$ in S. decipiens and 63.6\% in S. erinaceieuropaei. Two major non-coding regions present in 3 Spirometra species mtDNA were predicted between $\operatorname{trn} Y$ and $\operatorname{trnL1}$, and between $\operatorname{trnR}$ and nad5. Non-coding region 1 (NR1) between trnY and trnL1, was 204 nt (S. ranarum), 204 nt (S. decipiens) and 201 nt (S. erinaceieuropaei) in length, while non-coding region 2 (NR2) between trnR and nad5 was $175 \mathrm{nt}$ (S. ranarum), $175 \mathrm{nt}$ (S. decipiens) and $185 \mathrm{nt}$ (S. erinaceieuropaei) in length.

A percentage pairwise comparison of sequence divergence of the 12 protein-coding genes among S. ranarum, S. decipiens, and $S$. erinaceieuropaei is shown in Table 2 . The 12 proteincoding genes constituted 10,067 bp and 3,355 codons ( $S$. ranarum), 10,067 bp and 3,355 codons (S. decipiens), and 10,065 bp and 3,355 codons (S. erinaceieuropaei) of their respective mitochondrial genomes. The overall nucleotide sequence divergence of 12 protein-coding genes of $S$. ranarum and $S$. decipiens differed by $1.5 \%$, while that of $S$. ranarum and S. erinaceieuropaei differed by $13.0 \%$. The divergence of amino acid sequences of 12 protein-coding genes of $S$. ranarum and $S$. decipiens ranged from as low as $0.0 \%$ ( $\operatorname{cox} 2$ and nad6) to as high as $1.4 \%$ (nad4) (Table 2). The nucleotide sequence divergence of the nad4 gene (the most variable gene), and the cox 2 and nad6 (the most highly conserved gene) genes was $2.6 \%$ and $0.0 \%$ between $S$. ranarum and S. decipiens, respectively. The ribosomal RNA genes of $S$. ranarum and $S$. decipiens differed by $1.6 \%$ (16S rRNA) and 1\% (12S rRNA). The percent of nucleotide sequence difference was greater than that of the amino acid difference for the cox1, cox3, cob, atp6, nad1, nad2, nad3, nad4, and nad4L genes of S. ranarum and S. decipiens, suggesting the existence of synonymous substitutions.

The degree of divergence in mtDNA sequences between sister species or congeneric species was estimated using the genetic distance of the cob gene among mammalian groups such as amphibians, reptiles and avian birds. The sequence divergence of the sister or congeneric species cob gene among mammalian groups is greater than $2 \%$ [22]. Another study reported that cox1 divergence among 13,320 species in 11 animal phyla ranged from as low as $0.0 \%$ to as high as $53.7 \%$, while $79 \%$ of those species showed greater than $8 \%$ sequence divergence at the species taxonomy level [23]. The aforementioned studies have established that $98 \%$ of congeneric animal species showed greater than $2 \%$ sequence divergence in the cox 1 gene $[22,23]$.

Mitochondrial cox1 sequence variation of Spirometra species has been reported to range from 0.0-3.5\% in China, Myanmar, Thailand, and Lao PDR, [24] and range from 0.0-2.6\% in Japan, India and Indonesia [25]. In the present study, the complete mitochondrial genome of $S$. ranarum was sequenced and characterized, and comparison with that of S. erinaceieuropaei and $S$. decipiens showed that overall nucleotide sequence divergence of 12 protein-coding genes of $S$. ranarum and S. decipiens differed by $1.5 \%$, with $100 \%$ sequence similarity in the cox 2 and nad6 genes, while the DNA sequence divergence of cox1, nad1, and nad4 genes of S. ranarum and S. decipiens was 2.2\%, $2.1 \%$ and $2.6 \%$, respectively. These cox1, nad1, and nad4 genes 
have been used as genetic markers for taxonomic identification and phylogenetic reconstruction by many researchers $[4,6-9,11$ $14,24,25]$. The degree of sequence divergence of the cox1, cob, nad1, and nad4 genes of $S$. ranarum and $S$. decipiens was greater than $2 \%$, which indicates that species are independent species. However, sequence differences of the cox3, atp6, nad2, nad3, nad4L, and nad5 genes of $S$. ranarum and $S$. decipiens was less than $2 \%$, while that of the cox 2 and nad 6 genes was $0 \%$, despite these 2 Spirometra species having morphological differences.

Although mitochondrial DNA sequence divergence has been useful as a genetic marker for species identification, including identification of sister or congeneric species, improving the reliability of this method requires the accumulation of DNA sequence data and criteria pertaining to morphological identification. A consensus has yet to be reached regarding criteria of mitochondrial DNA sequence divergence for species level taxonomy of Spirometra tapeworms, even though identified Spirometra species have been analyzed using mitochondrial DNA sequence divergence based on morphological characteristics. Further studies are needed to clarify species identification and to delineate the precise genetic variation of these Spirometra species.

\section{ACKNOWLEDGMENTS}

This work was supported by the National Research Foundation of Korea (No. 2017R1D1A3B03035976). The materials used were provided by the Parasite Resource Bank of Korea (PRB000720).

\section{CONFLICT OF INTEREST}

We have no conflict of interest related to this work.

\section{REFERENCES}

1. Faust EC, Campbell HE, Kellogg CR. Morphological and biological studies on the species of Diphyllobothrium in China. Am J Epidemiol 1929; 9: 560-583.

2. Wardle RA, McLeod JA. The Zoology of Tapeworms. Minneapolis, USA. University of Minnesota Press. 1952, pp 559-615.

3. A McIntosh. New host records for Diphyllobothrium mansonoides Mueller 1935. J Parasitol 1937; 23: 313-315.

4. Almeida GG, Coscarelli D, Melo MN, Melo AL, Pinto HA. Molecular identification of Spirometra spp. (Cestoda: Diphyllobothriidae) in some wild animals from Brazil. Parasitol Int 2016; 65: 428-431.

5. Baer JG. Contributions to the helminth fauna of South Africa.
Mammalian cestodes. Union S. Africa Dept Agric Rept Dir Vet Educ and Res 1926; 11: 1.

6. Jeon HK, Park HS, Lee DM, Choe SJ, Kim KH, Huh S, Sohn WM, Chai JY, Eom KS. Human infections with Spirometra decipiens plerocercoids identified by morphologic and genetic analyses in Korea. Korean J Parasitol 2015; 53: 299-305.

7. Jeon HK, Park HS, Lee DM, Choe SJ, YS Kang, Bia MM, Lee SH, Sohn WM, Hong SJ, Chai JY, Eom KS. Genetic and morphologic identification of Spirometra ranarum in Myanmar. Korean J Parasitol 2018; 56: 275-280.

8. Eom KS, Park HS, Lee DM, Choe SJ, YS Kang, Bia MM, Lee SH, Keyyu J, Fymagwa R, Jeon HK. Molecular and morphologic identification of Spirometra ranarum found in the stool of African lion, Panthera leo in the Serengeti plain of Tanzania. Korean J Parasitol 2018; 56: 379-383.

9. Jeon HK, Huh S, Sohn WM, Chai JY, Eom KS. Molecular genetic findings of Spirometra decipiens and S. ranarum in Korea. Korean J Parasitol 2018; 56: 359-364.

10. Le TH, Blair D, McManus DP. Mitochondrial genomes of parasitic flatworms. Trends Parasitol 2002; 18: 206-213.

11. Liu W, Zhao GH, Tan MY, Zeng DL, Wang KZ, Yuan ZG, Lin RQ, Zhu XQ, Liu Y. Survey of Spirometra erinaceieuropaei spargana infection in the frog Rana nigromaculata of the Hunan Province of China. Vet Parasitol 2010; 173: 152-156.

12. Zhang X, Cui J, Wei T, Li LY, Jiang J, Lu JC, Jiang P, Liu LN, Wang ZQ. Survey and genetic variation of Spirometra erinaceieuropaei sparganum in frogs and snakes from Guangxi of southern China. Trop Biomed 2014; 31: 862-870.

13. Zhang $\mathrm{X}$, Wang $\mathrm{H}$, Cui J, Jiang $\mathrm{P}$, Lin ML, Zhang YL, Liu RD, Wang ZQ. The phylogenetic diversity of Spirometra erinaceieuropaei isolates from southwest China revealed by multi genes. Acta Trop 2016; 156: 108-114.

14. Zhang X, Duan JY, Wang ZQ, Jiang P, Liu RD, Cui J. Using the small subunit of nuclear ribosomal DNA to reveal the phylogenetic position of the plerocercoid larvae of Spirometra tapeworms. Exp Parasitol 2017; 175: 1-7.

15. Park JK, Kim KH, Kang SH, Jeon HK, Kim JH, Littlewood DTJ, Eom KS. Characterization of the mitochondrial genome of Diphyllobothrium latum (Cestoda: Pseudophyllidea) implication for the phylogeny of eucestodes. Parasitol 2007; 134: 749-759.

16. Kim KH, Jeon HK, Kang S, Sultana T, Kim GJ, Eom KS, Park JK. Characterization of the complete mitochondrial genome of $\mathrm{Di}$ phyllobothrium nihonkaiense (Diphyllobothriidae: Cestoda), and development of molecular markers for differentiating fish tapeworms. Mol Cell 2007; 23: 379-390.

17. Eom KS, Park HS, Lee DM, Choe SJ, Kim KH, Jeon HK. Mitochondrial genome sequences of Spirometra erinaceieuropaei and $S$. decipiens (Cestoidea: Diphyllobothriidae). Korean J Parasitol 2015; 53: 455-463.

18. Bernt M, Donath A Jühling F, Externbrink F, Florentz C, Fritzsch G, Pütz J, Middendorf M, Stadler PF. MITOS: improved de novo metazoan mitochondrial genome annotation. Mol Phylogenet Evol 2013; 69: 313-319. 
19. Wyman SK, Jansen RK, Boore JL. Automatic annotation of organellar genomes with DOGMA. Bioinformatics 2004; 20:32523255.

20. Lowe TM, Eddy SR. tRNAscan-SE: a program improved detection of transfer DNA genes in genomic sequence. Nucleic Acids Res 1997; 25: 955-964.

21. Matzura O, Wennborg A. RNAdraw: an integrated program for RNA secondary structure calculation and analysis under 32-bit Microsoft Windows. Comput Appl Biosci 1996; 12: 247-249.

22. Johns GC, Avise JC. A comparative summary of genetic distances in the vertebrates from the mitochondrial cytochrome $\mathrm{b}$ gene. Mol Bio Evol 1998; 15: 1481-1490.

23. Herbert PDN, Ratnasingham S, deWaard JR. Barcoding animal life: cytochrome c oxidase subunit 1 divergences among closely related species. Proc Biol Sci 2003; 270 (suppl): 96-99.

24. Jongthawin J, Intapan PM, Sanpool O, Sadaow L, Laymanivong S, Thanchomnang T, Maleewong W. Molecular evidence of Spirometra erinaceieuropaei infection in snakes Ptyas korros from Lao PDR and Thailand and frogs Hoplobatrachus rugulosus from Myanmar. Southeast Asian J Trop Med Public Health 2014; 45: 1271-1278.

25. Okamoto M, Iseto C, Shibahara T, Sato MO, Wandra T, Craig PS, Ito A. Intraspecific variation of Spirometra erinaceieuropaei and phylogenetic relationship between Spirometra and Diphyllobothrium inferred from mitochondrial CO1 gene sequence. Parasitol Int 2007; 56: 235-238. 